\title{
Longitudinal Associations of Body Mass Index, Waist Circumference, and Waist-to-Hip Ratio with Biomarkers of Oxidative Stress in Older Adults: Results of a Large Cohort Study
}

\author{
Ankita Anusrutia, ${ }^{a}$ Eugène H.J.M. Jansen ${ }^{c} \quad$ Xīn Gào $^{a, b} \quad$ Yang Xuan ${ }^{a, b}$ \\ Hermann Brenner ${ }^{a, b}, d, e$ Ben Schöttker ${ }^{a, b}$ \\ aDivision of Clinical Epidemiology and Aging Research, German Cancer Research Center \\ (DKFZ), Heidelberg, Germany; ${ }^{b}$ Network Aging Research, Heidelberg University, Heidelberg, \\ Germany; ${ }^{c}$ Center for Health Protection, National Institute of Public Health and the \\ Environment, Bilthoven, The Netherlands; ${ }^{\mathrm{d}}$ German Cancer Consortium (DKTK), German \\ Cancer Research Center (DKFZ), Heidelberg, Germany; ${ }^{e}$ Division of Preventive Oncology, \\ German Cancer Research Center (DKFZ) and National Center for Tumor Diseases (NCT), \\ Heidelberg, Germany
}

\section{Keywords}

Body mass index - Waist circumference - Waist-to-hip ratio - Oxidative stress - Free radicals . Reactive oxygen metabolites $\cdot$ Total thiol levels

\begin{abstract}
Background: In the literature, obesity is discussed as a determinant of high oxidative stress (OS). Hence, prevention or reduction of obesity could prevent high OS and subsequently serve as a target for "healthy aging." Methods: Diacron's reactive oxygen metabolites test (D-ROM) and total thiol levels (TTL), a marker of antioxidant defense capacity, were measured in 1,734 participants of a population-based cohort study of older adults (age range: 57-83 years) at 2 time points 3 years apart. The longitudinal associations of body mass index, waist-to-hip ratio, and waist circumference with D-ROM and TTL were assessed with multivariable adjusted generalized linear models. Dose-response analyses were conducted with restricted cubic splines. Results: D-ROM was not significantly associated with any of the weight measures. On the contrary, TTL showed statistically significant, inverse linear associations with all weight measures. Conclusion: A healthy body weight seems to be highly relevant for the antioxidative defense capacity of human beings. In contrast, D-ROM levels were independent of the study participant's weight. Clinical trials are needed to corroborate if loss of weight by obese individuals can effectively increase TTL and subsequently also life expectancy.
\end{abstract}




\section{Introduction}

The free radical theory, which postulates that reactive oxygen species (ROS) lead to accelerated aging, proposed by Harman [1] has attracted increased attention. Any imbalance between ROS and antioxidants at a cellular level triggers oxidative stress (OS) [2], which may disrupt normal cell function due to oxidative damage to lipids, proteins, and DNA [3] and, hence, contributes to cell senescence. Subsequently, it can be inferred that scaling up the antioxidant defense mechanisms and/or scaling down ROS damage could reduce OS and, thereby, delay the process of aging [2]. In addition to glutathione, thiol groups of proteins can be oxidized reversibly and help to control redox processes [4]. Therefore, total thiol levels (TTL) can be regarded as an indirect serum biomarker for the antioxidant defense capacity of cells. ROS damage can be indirectly measured by the serum biomarker “Diacron's reactive oxygen metabolites test" (D-ROM), which is mainly a biomarker of lipid peroxidation. D-ROM and TTL have been shown to be very stable in blood samples stored for many years [5]. Both high D-ROM levels and low TTL have been documented to be associated with OS-related diseases [6] and mortality [7].

In the literature, obesity is being discussed as a major determinant of high OS [8]. It has been postulated to escalate OS, which in turn can induce related metabolic conditions [9]. Hence, prevention or reduction of obesity could prevent high OS [10] and subsequently serve as a target for "healthy aging." Several cross-sectional studies have detected an association or correlation of OS biomarkers and body mass index (BMI) [11-13]. However, the directionality of the association of obesity and OS has been argued upon [14]. For a causal relationship, weight measures need to predict future OS in a longitudinal study design. To our knowledge, there have been no population-based cohort studies investigating longitudinal associations of various anthropometric measures with OS biomarkers so far. Therefore, we conducted the first longitudinal study on various weight measures and their associations with established OS biomarkers. In detail, we aimed at investigating whether BMI, waist circumference (WC), and waist-to-hip ratio (WHR) are associated longitudinally with D-ROM and TTL serum concentrations in a large cohort of older adults (age range: 57-83 years) as this could be an avenue to "healthy aging."

\section{Materials and Methods}

\section{Study Design}

The analyses were conducted in the ESTHER study (Epidemiological Study on the Chances in the Prevention, Early Recognition, and Optimized Treatment of Chronic Diseases in the Older Population/Epidemiologische Studie zu Chancen der Verhütung, Früherkennung und optimierten Therapie chronischer Erkrankungen in der älteren Bevölkerung), an ongoing cohort study of older adults living in the Saarland, a state located in Southwest Germany [15]. The study was approved by the Ethics Committees of the Medical Faculty of the University of Heidelberg and the Medical Association of Saarland, and was conducted in accordance with the Declaration of Helsinki. The total number of participants amounted to 9,940 adults between 50 and 75 years of age, who were all recruited by their respective general practitioners (GPs) in the context of a routine health examination between 2000 and 2002. Follow-up (FU) assessments by self-administered questionnaires were conducted 2, 5, 8, 11, and 14 years after baseline, and the study participant's GP filled out questionnaires about the participant's medical conditions. Blood samples were collected at the 5-, 8-, 11-, and 14-year FU. Additionally, participants were offered a 3-h home visit at the 8-, 11-, and 14-year FU, which included detailed geriatric, pharmacological, and anthropometric assessments by study physicians. 
Fig. 1. Flowchart of ESTHER study participants. BMI, body mass index; D-ROM, Diacron's reactive oxygen metabolites test; TTL, total thiol levels; WC, waist circumference; WHR, waist-tohip ratio. ${ }^{a}$ High hemolytic, icteric, or lipemic index.

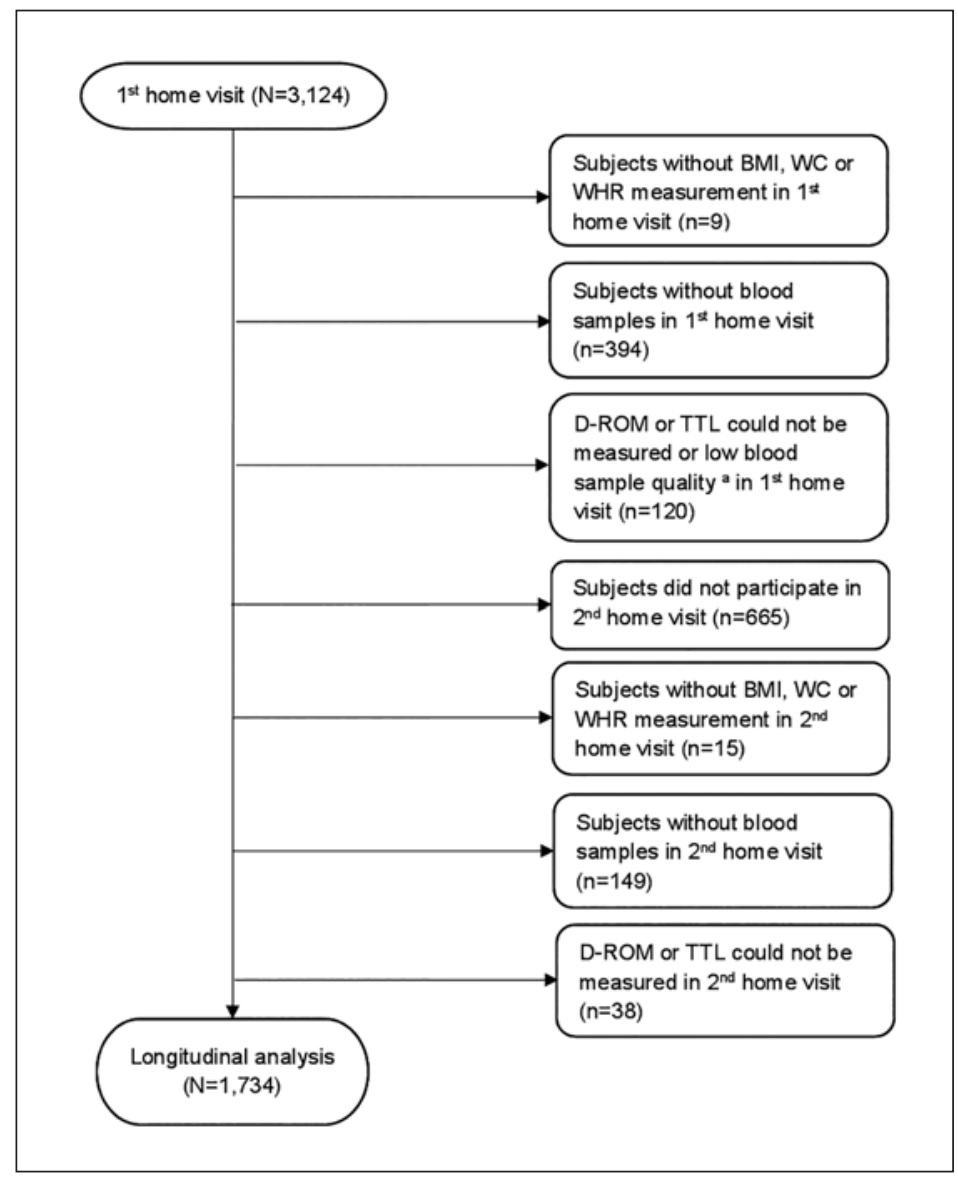

\section{Inclusion and Exclusion Criteria}

The 1st home visit (performed at the 8-year FU of the ESTHER study from 2008 to 2010) furnished as the baseline and the 2nd home visit (performed at the 11-year FU of the ESTHER study from 2011 to 2013) was used as the FU for the current investigation; 3,124 study participants were followed up at the 1st home visit, and the allocation of the study population is depicted in Figure 1. We excluded participants without anthropometric measurements, missing blood samples, or missing D-ROM and TTL measurements at the 1st and/or 2nd home visit. Furthermore, 665 study participants were excluded because they did not take part at the 2 nd home visit. In summary, the final sample size for the longitudinal analysis was 1,734 subjects.

\section{Predictors, Outcomes, and Covariates}

The predictors of interest comprised BMI, WC, and WHR. Height, weight, WC, and hip circumference were measured by the study physicians during the physical examination at the home visits. The following cutoffs of the different measures were used for categorical variable definitions. For BMI, WHO cutoffs were used $\left(<25,25-<30,30-<35\right.$, and $\left.\geq 35 \mathrm{~kg} / \mathrm{m}^{2}\right)[16]$, with the exception that the lowest category was not coded because very few of the elderly study participants had BMI $<18.5 \mathrm{~kg} / \mathrm{m}^{2}(n=5)$. For WC, the cutoffs of $\geq 102 \mathrm{~cm}$ in men and $\geq 88 \mathrm{~cm}$ in women were applied to define obesity [17], and for WHR, the cutoffs were placed at $\geq 0.90$ for males and $\geq 0.85$ for females for an obesity definition [18].

The outcomes of interest were changes in D-ROM and TTL concentrations between the 1 st and the 2 nd home visit for a prospective analysis. The assays used to measure D-ROM 
(Diacron, Grosseto, Italy) and TTL (Rel Assay Diagnostics, Gaziantep, Turkey) were adapted to an autoanalyzer (LX20-Pro; Beckman-Coulter, Woerden, The Netherlands), and measurements were performed in serum samples at the Laboratory for Health Protection Research (Bilthoven, The Netherlands) [19].

Other biomarkers that served as covariates were analyzed from serum samples in a central laboratory (Synlab, Heidelberg, Germany). Total cholesterol and high-density cholesterol (HDL) were assessed by enzymatic colorimetric tests (analytes: Chol2 2100 and HDLC3 450; Roche Diagnostics, Mannheim, Germany) and C-reactive protein (CRP) by immunoturbidimetry on a cobas 8000 C701 (analyte: CRPL3 500, Roche Diagnostics, Mannheim, Germany). Information on sociodemographic factors (age, sex, and educational status) and lifestyle factors (smoking and alcohol consumption) were taken from the participant's questionnaire, and information on medical history (diabetes mellitus, hypertension, and history of stroke, myocardial infarction, and cancer) were taken from the GPs questionnaires. Subjects with diabetes mellitus or hypertension were additionally recorded when the GP or study participant reported drug use against these chronic diseases. Physical activity was assessed via interview at the home visits with a questionnaire instrument specifically developed for older adults by Voorrips et al. [20].

\section{Statistical Analysis}

Spearman correlation coefficients $(r)$ were calculated to assess correlations between weight measures, D-ROM levels, and TTL at the 1st home visit. Correlation coefficients were interpreted as negligible $(|r|<0.3)$, low $(|r| 0.3$ to $<0.5)$, moderate $(|r| 0.5$ to $<0.7)$, high ( $|r| 0.7$ to $<0.9$ ), or very high $(|r| 0.9$ to 1.0 ) correlation applying commonly used cutoffs in medical science [21]. Generalized linear models were used to assess the association of both continuously (estimates per 1 standard deviation [SD]) and categorically modelled BMI, WC, and WHR with changes $(\Delta)$ in D-ROM and TTL concentrations observed between the 1st and the 2nd home visit. In order to investigate the shape of the associations, dose-response modeling with the restricted cubic splines SAS macro of Desquilbet and Mariotti [22] was applied. Five knots where specified at the 5th, 25th, 50th, 75th, and 95th percentiles of the BMI, WC, or WHR distribution while using the 5 th percentile as the reference.

All models were adjusted for age, sex, years of school education, alcohol consumption, number of cigarettes smoked per day, physical activity, the baseline values of D-ROM or TTL, and variables for the change in age, alcohol consumption, daily cigarette consumption, and physical activity from the 1 st to the 2 nd home visit. In a sensitivity model, further covariates were added that could also be intermediates in the pathway from increased weight to OS: Total cholesterol, HDL cholesterol, CRP, and a history of cancer, diabetes mellitus, hypertension, myocardial infarction, or stroke as well as variables for changes in these covariates from the 1 st to the 2 nd home visit. In a further sensitivity analysis, study participants who lost $5 \mathrm{~kg}$ of weight or more involuntarily in the year before the 2 nd home visit were excluded. Involuntary weight loss can be a symptom of undiagnosed cancer and could confound the results because cancer is associated with weight loss and increased OS [23]. Furthermore, sex-stratified analyses were performed with respect to the differences in body composition between males and females regarding fat distribution [24]. In addition, statistical tests on interactions of sex and anthropometric measures were carried out.

Statistical analyses were carried out with SAS 9.4, (Cary, NC, USA), and all tests were performed two sided using an $\alpha$-level of 0.05 . Multiple imputation of 5 data sets was undertaken to deal with missing values. The proportion of missing values was not higher than $2 \%$ for all variables (Table 1) and missingness at random could be assumed. If not stated otherwise, results of imputed datasets were combined by the SAS procedure PROC MIANALYZE. 
Table 1. Characteristics of the study participants at the 1st and the 2nd home visit of this research project (ESTHER study, $n=1,734$ )

\begin{tabular}{|c|c|c|c|c|c|c|}
\hline \multirow[t]{2}{*}{ Characteristics } & \multicolumn{3}{|c|}{ 1st home visit } & \multicolumn{3}{|c|}{ 2nd home visit } \\
\hline & total $n$ & $n(\%)$ & mean (SD) & total $n$ & $n(\%)$ & mean (SD) \\
\hline Age, years & 1,733 & - & $68.4(6.2)$ & 1,733 & - & $71.2(6.1)$ \\
\hline Males & 1,734 & $832(48.0)$ & - & 1,734 & $832(48.0)$ & - \\
\hline School education & 1,710 & & & 1,710 & & \\
\hline$\leq 9$ years & & $1,114(65.1)$ & - & & $1,114(65.1)$ & - \\
\hline $10-11$ years & & $312(18.3)$ & - & & $312(18.3)$ & - \\
\hline$\geq 12$ years & & $284(16.6)$ & - & & $284(16.6)$ & - \\
\hline Smoking & 1,730 & & & & & \\
\hline Nonsmokers & & $1,615(93.4)$ & - & & $1,592(93.9)$ & - \\
\hline Current smokers & & $115(6.6)$ & - & & $103(6.1)$ & - \\
\hline Cigarettes smoked/day & 115 & - & $16.4(16.1)$ & 103 & - & $14.8(10.5)$ \\
\hline Alcohol consumption & 1,704 & & & 1,670 & & \\
\hline Nonconsumers & & $456(26.8)$ & - & & $464(27.8)$ & - \\
\hline Consumers & & $1,248(73.2)$ & - & & $1,206(72.2)$ & - \\
\hline Physical activity (no unit) ${ }^{\mathrm{a}}$ & 1,733 & - & $18.7(9.9)$ & 1,734 & - & $14.3(9.2)$ \\
\hline Total cholesterol, mg/dL & 1,734 & - & $235(47.0)$ & 1,726 & - & $209.4(43.5)$ \\
\hline HDL cholesterol, mg/dL & 1,733 & - & $60.1(15.2)$ & 1,726 & - & $59.3(16.7)$ \\
\hline C-reactive protein, mg/L & 1,733 & - & $3.3(6.7)$ & 1,733 & - & $3.6(8.7)$ \\
\hline Hypertension & 1,734 & $1,205(69.5)$ & - & 1,733 & $1,356(78.3)$ & - \\
\hline Diabetes mellitus & 1,725 & 340 (19.7) & - & 1,732 & $484(27.9)$ & - \\
\hline History of cancer & 1,725 & $204(11.8)$ & - & 1,732 & $292(16.9)$ & - \\
\hline History of stroke & 1,725 & $124(7.2)$ & - & 1,732 & $220(12.7)$ & - \\
\hline History of myocardial infarction & 1,725 & $102(5.9)$ & - & 1,732 & $135(7.8)$ & - \\
\hline Body mass index (BMI), $\mathrm{kg} / \mathrm{m}^{2}$ & 1,734 & - & $28.6(4.7)$ & 1,734 & - & $28.4(4.7)$ \\
\hline BMI category & 1,734 & & & 1,734 & & \\
\hline$<25 \mathrm{~kg} / \mathrm{m}^{2}$ & & $370(21.4)$ & - & & $388(22.4)$ & - \\
\hline$\geq 25-<30 \mathrm{~kg} / \mathrm{m}^{2}$ & & 775 (44.7) & - & & $782(45.1)$ & - \\
\hline$\geq 30-<35 \mathrm{~kg} / \mathrm{m}^{2}$ & & $429(24.7)$ & - & & $418(24.1)$ & - \\
\hline$\geq 35 \mathrm{~kg} / \mathrm{m}^{2}$ & & $160(9.2)$ & - & & $146(8.4)$ & - \\
\hline $\mathrm{WC}, \mathrm{cm}$ & 1,734 & - & $97.2(13.1)$ & 1,734 & - & $96.6(13.3)$ \\
\hline Obesity by $\mathrm{WC}^{\mathrm{c}}$ & 1,734 & & & 1,734 & & \\
\hline Nonobese & & $729(42.0)$ & - & & $772(44.5)$ & - \\
\hline Obese & & $1,005(58.0)$ & - & & $962(55.5)$ & - \\
\hline WHR (no unit) & 1,734 & - & $0.9(0.1)$ & 1,734 & - & $0.9(0.1)$ \\
\hline Obesity by WHR ${ }^{\mathrm{d}}$ & 1,734 & & & 1,734 & & \\
\hline Nonobese & & 477 (27.5) & - & & 496 (28.6) & - \\
\hline Obese & & $1,257(72.5)$ & - & & $1,238(71.4)$ & - \\
\hline D-ROM, Carr unit & 1,734 & - & 348 (71) & & - & 349 (59) \\
\hline $\mathrm{TTL}, \mu \mathrm{mol} / \mathrm{L}$ & 1,734 & - & $328(75)$ & & - & $279(71)$ \\
\hline
\end{tabular}

D-ROM; Diacron's reactive oxygen metabolites test; SD, standard deviation; TTL, total thiol levels; WC, waist circumference; WHR, waist-to-hip ratio. Total numbers of participants evaluated are also listed.

${ }^{a}$ Total score in the physical activity questionnaire for the elderly by Voorrips et al. [20]. ${ }^{\mathrm{b}}$ New cases observed between the 8- and the 11-year follow-up home visit. ${ }^{\mathrm{c}}$ Definition of obesity by WC: $\geq 102 \mathrm{~cm}$ in men or $\geq 88 \mathrm{~cm}$ in women [17]. ${ }^{\mathrm{d}}$ Definition of obesity by WHR: $\geq 0.90$ for males and $\geq 0.85$ for females [18].

\section{Results}

The characteristics of the 1,734 study participants included in the 1st and the 2nd home visit are shown in Table 1. At the 1st home visit, their mean age was 68.4 years (age range: 57-83 years), and slightly more men than women (48\%) were included. The prevalence rates of obesity by BMI $\left(>30 \mathrm{~kg} / \mathrm{m}^{2}\right), \mathrm{WC}$, and WHR, were $33.9,58.0$, and $72.5 \%$, respectively. The 
Table 2. Spearman correlation coefficients (upper rows) with $p$ values (lower rows) between weight measures, D-ROM, and TTL measured at the 1 st home visit (ESTHER study, $n=1,734$ )

\begin{tabular}{lrrrrc}
\hline & BMI & WC & WHR & \multicolumn{1}{c}{ D-ROM } & \multicolumn{1}{c}{ TTL } \\
\hline BMI & & 0.760 & 0.346 & 0.009 & -0.173 \\
& & $<0.001$ & $<0.001$ & 0.399 & $<0.001$ \\
\hline WC & 0.760 & & 0.740 & -0.141 & -0.114 \\
& $<0.001$ & & $<0.001$ & $<0.001$ & $<0.001$ \\
\hline WHR & 0.346 & 0.740 & & -0.278 & 0.004 \\
& $<0.001$ & $<0.001$ & & $<0.001$ & 0.718 \\
\hline D-ROM & 0.009 & -0.141 & -0.278 & & -0.001 \\
& 0.399 & $<0.001$ & $<0.001$ & & 0.9418 \\
\hline TTL & -0.173 & -0.114 & 0.004 & -0.001 & \\
& $<0.001$ & $<0.001$ & 0.718 & 0.942 & \\
\hline
\end{tabular}

D-ROM, Diacron's reactive oxygen metabolites test; TTL, total thiol levels; WC, waist circumference; WHR, waist-to-hip ratio.

Table 3. Longitudinal associations of baseline weight measures with $\triangle \mathrm{D}-\mathrm{ROM}$ and $\Delta \mathrm{TTL}$ concentrations in adjusted generalized linear models (ESTHER study, $n=1,734$ )

\begin{tabular}{|c|c|c|c|c|c|}
\hline \multirow[t]{2}{*}{ Characteristics } & \multirow[t]{2}{*}{ Total $n$} & \multicolumn{2}{|l|}{$\Delta \mathrm{D}-\mathrm{ROM}^{\mathrm{a}}$} & \multicolumn{2}{|l|}{$\Delta \mathrm{TTL}^{\mathrm{a}}$} \\
\hline & & estimate & $p$ value & estimate & $p$ value \\
\hline BMI $\left(/ 4.8 \mathrm{~kg} / \mathrm{m}^{2}\left[\mathrm{SD}^{]}\right)\right.$ & 1,734 & 0.321 & 0.788 & -10.723 & $<0.0001$ \\
\hline \multicolumn{6}{|l|}{ BMI categorically } \\
\hline$<25 \mathrm{~kg} / \mathrm{m}^{2}$ & 370 & Ref. & & Ref. & \\
\hline$\geq 25-<30 \mathrm{~kg} / \mathrm{m}^{2}$ & 775 & 0.650 & 0.833 & -15.738 & $<0.0001$ \\
\hline$\geq 30-<35 \mathrm{~kg} / \mathrm{m}^{2}$ & 429 & -3.141 & 0.367 & -26.489 & $<0.0001$ \\
\hline$\geq 35 \mathrm{~kg} / \mathrm{m}^{2}$ & 160 & 2.769 & 0.550 & -32.707 & $<0.0001$ \\
\hline WC (/13.4 cm [SD]) & 1,734 & 0.859 & 0.513 & -10.916 & $<0.0001$ \\
\hline \multicolumn{6}{|l|}{ WC categorically ${ }^{\mathrm{b}}$} \\
\hline Nonobese & 729 & Ref. & & Ref. & \\
\hline Obese & 1,005 & -0.157 & 0.948 & -15.066 & $<0.0001$ \\
\hline WHR (/0.1 [SD]) & 1,734 & 1.430 & 0.349 & -7.512 & 0.0003 \\
\hline \multicolumn{6}{|l|}{ WHR categoricallyc } \\
\hline Nonobese & 477 & Ref. & & Ref. & \\
\hline Obese & 1,257 & -0.057 & 0.984 & -3.651 & 0.319 \\
\hline
\end{tabular}

Statistically significant results are in bold. See the text or the legend to Table 1 for abbreviations.

${ }^{a}$ All models are adjusted for baseline age, sex, education, alcohol consumption, daily cigarette consumption, physical activity, the respective oxidative stress marker values at baseline and variables for the change in age, alcohol consumption, daily cigarette consumption, and physical activity from the 1 st to the 2 nd home visit. ${ }^{b}$ Definition of obesity by WC: $\geq 102 \mathrm{~cm}$ in men or $\geq 88 \mathrm{~cm}$ in women [17]. ${ }^{\mathrm{c}}$ Definition of obesity by WHR: $\geq 0.90$ for males and $\geq 0.85$ for females [18].

mean BMI, WC, WHR, and D-ROM levels remained fairly stable in the 3 years from the 1st to the 2 nd home visit, and all other covariables were also subject to little changes. However, TTL was an exception with a strong decrease in mean TTL by $49 \mu \mathrm{mol} / \mathrm{L}$.

The correlation matrix (Table 2) shows that high correlations (all $r>0.7$ ) were observed between BMI and WC as well as between WHR and WC, whereas the correlation between BMI 


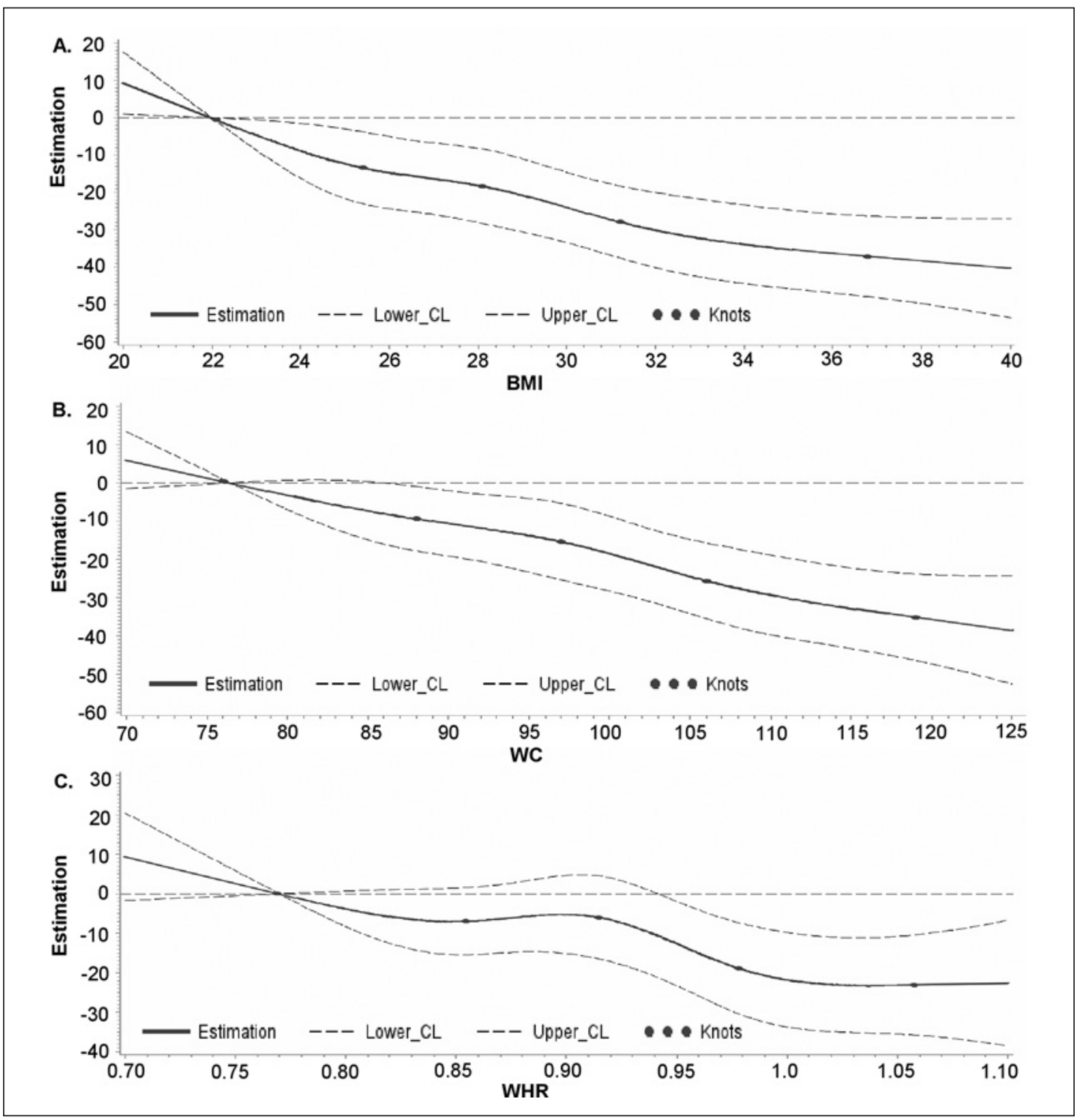

Fig. 2. Dose-response curve modeled with restricted cubic splines for the longitudinal association of body mass index (BMI; A), waist circumference (WC; B), and waist-to-hip ratio (WHR; C) with $\Delta$ total thiol levels (TTL). Lower_CL, lower confidence limit; Upper_CL, upper confidence limit. The model is adjusted for baseline age, sex, education, alcohol consumption, daily cigarette consumption, physical activity, TTL at baseline, and variables for the change in age, alcohol consumption, daily cigarette consumption, and physical activity from the 1 st to the 2 nd home visit. Note: the analysis was performed in the first of the 5 imputed data sets.

and WHR was rather low ( $r \approx 0.3$ at both home visits). However, D-ROM and TTL did not correlate with each other. Furthermore, negligible, albeit statistically significant correlation coefficients of $0.114 \leq|r| \leq 0.288$ were detected between the weight measures WC and WHR and D-ROM and between the weight measures BMI and WC and TTL. BMI was not correlated with D-ROM, and WHR was not correlated with TTL. D-ROM levels measured at the 1 st and 2 nd home visit correlated moderately with each other $(r=0.582)$, while the TTL measurements at the 1 st and the 2 nd home visit showed slightly lower correlations with each other $(r=0.428)$.

In multivariable adjusted generalized linear models, all 3 weight measures were not significantly associated with D-ROM (Table 3). However, all weight measures showed statis- 
Table 4. Sex-stratified results for female and male study participants for baseline weight measures with $\triangle \mathrm{D}$-ROM and $\Delta \mathrm{TTL}$ concentrations in adjusted generalized linear models (ESTHER study, $n=1,734$ )

\begin{tabular}{|c|c|c|c|c|c|c|c|c|}
\hline \multirow[t]{3}{*}{ Characteristics } & \multicolumn{4}{|l|}{$\Delta \mathrm{D}-\mathrm{ROM}^{\mathrm{a}}$} & \multicolumn{4}{|l|}{$\Delta \mathrm{TTL}^{\mathrm{a}}$} \\
\hline & \multicolumn{2}{|c|}{ female $(n=902)$} & \multicolumn{2}{|c|}{ male $(n=832)$} & \multicolumn{2}{|c|}{ female $(n=902)$} & \multicolumn{2}{|c|}{ male $(n=832)$} \\
\hline & estimate & $p$ value & estimate & $p$ value & estimate & $p$ value & estimate & $p$ value \\
\hline $\operatorname{BMI}\left(/ 5.2 \mathrm{~kg} / \mathrm{m}^{2}\left[\mathrm{SD}^{]}\right)\right.$ & 2.080 & 0.185 & -2.129 & 0.257 & -9.238 & $<0.0001$ & -13.344 & $<0.0001$ \\
\hline \multicolumn{9}{|l|}{ BMI categorically } \\
\hline$<25 \mathrm{~kg} / \mathrm{m}^{2}$ & Ref & & Ref & & Ref & & Ref & \\
\hline$\geq 25-<30 \mathrm{~kg} / \mathrm{m}^{2}$ & 4.675 & 0.255 & -5.802 & 0.220 & -17.097 & 0.0007 & -17.094 & 0.009 \\
\hline$\geq 30-<35 \mathrm{~kg} / \mathrm{m}^{2}$ & -1.982 & 0.680 & -6.832 & 0.189 & -25.606 & $<0.0001$ & -29.963 & $<0.0001$ \\
\hline$\geq 35 \mathrm{~kg} / \mathrm{m}^{2}$ & 12.028 & 0.045 & -12.244 & 0.098 & -28.428 & $<0.0001$ & -40.498 & $<0.0001$ \\
\hline $\mathrm{WC}(/ 13.1 \mathrm{~cm}[\mathrm{SD}])$ & 0.628 & 0.722 & 1.501 & 0.452 & -9.844 & $<0.0001$ & -12.355 & $<0.0001$ \\
\hline \multicolumn{9}{|l|}{ WC categorically ${ }^{\mathrm{b}}$} \\
\hline Nonobese & Ref & & Ref & & Ref & & Ref & \\
\hline Obese & -2.490 & 0.472 & 3.007 & 0.371 & -12.197 & 0.004 & -17.535 & 0.0002 \\
\hline WHR (/0.1 [SD]) & -0.406 & 0.840 & 5.023 & 0.038 & -5.0 & 0.0425 & -9.929 & 0.003 \\
\hline \multicolumn{9}{|l|}{ WHR categorically } \\
\hline Nonobese & Ref & & Ref & & Ref & & Ref & \\
\hline Obese & -0.866 & 0.798 & 3.371 & 0.529 & -3.239 & 0.434 & -3.673 & 0.624 \\
\hline
\end{tabular}

Statistically significant results are in bold. See the text or the legend to Table 1 for abbreviations.

a All models are adjusted for baseline age, education, alcohol consumption, daily cigarette consumption, physical activity, the respective oxidative stress marker values at baseline, and variables for the change in age, alcohol consumption, daily cigarette consumption, and physical activity from the 1 st to the 2 nd home visit. ${ }^{b}$ Definition of obesity by WC: $\geq 102 \mathrm{~cm}$ in men or $\geq 88 \mathrm{~cm}$ in women. ${ }^{\mathrm{c}}$ Definition of obesity by WHR: $\geq 0.90$ for males and $\geq 0.85$ for females.

tically significant inverse associations with TTL, with the exception that WHR was only statistically significant when modeled continuously. The dose-response curves showed inverse linear associations of BMI, WC, and WHR with TTL (Fig. 2). In sensitivity analyses, adjusting for additional covariates, such as CRP, total and HDL cholesterol, and diseases, or excluding study participants who lost $5 \mathrm{~kg}$ of weight or more involuntarily in the year before the $2 \mathrm{nd}$ home visit, the results were not substantially different compared to the main results (data not shown).

Analyses stratified by sex yielded results similar to the results in the total sample for $\triangle \mathrm{TTL}$, speaking against a sex difference for this biomarker (Table 4). However, 2 divergent results for D-ROM were observed in males and females. First, BMI $\geq 35 \mathrm{~kg} / \mathrm{m}^{2}$ was statistically significantly associated with $\triangle \mathrm{D}-\mathrm{ROM}$ in females but not in males. This sex difference was confirmed by including an interaction term of sex and BMI $\geq 35 \mathrm{~kg} / \mathrm{m}^{2}$ versus BMI $<25 \mathrm{~kg} / \mathrm{m}^{2}$ in the model, which was statistically significantly associated with $\triangle \mathrm{D}-\mathrm{ROM}(p=0.02)$. Second, continuously modelled WHR was statistically significantly associated with $\triangle \mathrm{D}-\mathrm{ROM}$ in males but not in females, and the interaction term was close to statistical significance $(p=0.06)$. However, these sex differences for $\triangle \mathrm{D}$-ROM were not consistently observed for all weight measures and could be random findings because of multiple testing.

\section{Discussion}

In this large cohort study of older adults, BMI, WC, and WHR were not associated with D-ROM levels. On the contrary, all weight measures were statistically significantly, inverse linearly associated with TTL. The null result for D-ROM in our study is not supported by other studies, which used urinary 8-epi-prostaglandin F2 $\alpha$ (8-epi-PGF2 $\alpha$ ) to measure lipid peroxi- 
dation $[11,25]$. In the Framingham study, 8-epi-PGF2 $\alpha$ was associated with the BMI [11], and in a Japanese cohort of older adults, 8-epi-PGF2 $\alpha$ was correlated with the visceral fat area [25]. As 8-epi-PGF2 $\alpha$ is considered the most reliable biomarker of OS [26], we do not doubt the results of the cited studies and rather think that our study with D-ROM lacked statistical power.

However, our results for TTL, a proxy for the antioxidative capacity of tissues, are in agreement with previous cross-sectional studies, which have linked abdominal obesity to a decreased antioxidant capacity [27-29]. We add to this evidence by presenting the first longitudinal analysis to address the association of weight measures with TTL and observed an inverse association between BMI, WC, and WHR with TTL. Associations with TTL were comparably strong for BMI and WC, which were also highly correlated in our study sample. Results for WHR were a little weaker, which was in line with a lower correlation between BMI and WHR. This points into the direction that both general body fatness (best measured by BMI [30]) and visceral fat mass (best measured by WC [31]) have a relevant association with TTL.

This longitudinal association speaks for a causal relationship, and there is biological evidence that links visceral fat to a decreased antioxidant capacity of tissues. Decreased activity of antioxidant enzymes was successfully demonstrated in white adipose tissue of visceral fat within the accumulated fat of mouse models [10]. White adipose tissue is regarded as an endocrine organ and can produce adipocytokines [32]. Additionally, proinflammatory cytokines like TNF- $\alpha$, IL-1, and IL- 6 are produced by visceral body fat. These, in turn, augment OS by increased generation of ROS and nitrogen [33]. Taken together, this endocrine function of accumulated visceral body fat is an important source for systemic OS and subsequently decreased antioxidant capacities. Nevertheless, further studies are warranted to find the exact mechanisms that lower the redox defense mechanisms in obese individuals. In addition, a randomized clinical trial would be helpful to corroborate our findings. Such a trial could have great public health relevance because TTL decreases with age, which we also detected in a previous analysis of our study [7]. An effective measure that can reduce the age-related TTL decrease could be a measure for healthy aging because TTL was shown to be associated with cardiovascular mortality [7]. These thoughts are in line with the accumulating evidence that caloric restriction may increase longevity [34].

The major strength of our study is its prospective design, which we achieved by considering repeated measurements at 2 time points. However, it would have further strengthened the results and statistical power if we could have included more time points with repeated measurements in our study. Another advantage was the large sample size of our study, which enhanced the statistical power. The most important limitation of our study is its observational design. Although we adjusted for all available potential confounders in our study, residual confounding by factors we did not adjust for cannot be fully excluded. Finally, we would like to state that the study results can only be generalized to older Caucasian adults, aged 57-83 years, i.e. the participants of our study sample.

\section{Conclusion}

In conclusion, this first longitudinal analysis of weight measures and OS biomarkers supports the hypotheses that body fat measures are relevantly associated with a decreased antioxidant defense capacity of the organism. We showed that these associations are inversely linear in older adults (among whom almost no one was anorectic), which suggests the rule: the leaner the individual, the better the antioxidant defense capacity. Clinical trials are needed to corroborate if weight reduction in obese, older adults can effectively increase TTL and subsequently increase life expectancy. 


\section{Acknowledgment}

We extend our gratitude to all ESTHER study participants and their GPs.

\section{Statement of Ethics}

Participants have given their written informed consent. The study was approved by the Ethics Committees of Medical Faculty of the University of Heidelberg and the Medical Association of Saarland, and was conducted in line with the Declaration of Helsinki.

\section{Disclosure Statement}

The authors have no conflicts of interest to declare.

\section{Funding Sources}

This study was funded by a grant from the German Research Foundation (grant No. SCHO 1545/3-1). The ESTHER study was funded by grants from the Ministry for Social Affairs, Health, Women, and Family Affairs of the Saarland (Saarbrücken, Germany), the Ministry of Science, Research, and Arts of Baden-Württemberg (Stuttgart, Germany), the Federal Ministry of Education and Research (Berlin, Germany), and the Federal Ministry of Family Affairs, Senior Citizens, Women, and Youth (Berlin, Germany). The funders were not involved in any way in this project - neither study design, execution, analysis, data interpretation, nor writing of the manuscript.

\section{Author Contributions}

A.A. conducted the statistical analyses. A.A. wrote the first draft of the paper, and B.S. edited it. E.H.J.M. contributed to the measurement of the OS biomarkers. H.B., X.G., and Y.X. reviewed the draft of the manuscript and contributed to the interpretation of the results and the discussion.

\section{References}

1 Harman D. Aging: a theory based on free radical and radiation chemistry. J Gerontol. 1956 Jul;11(3):298-300.

2 Salmon AB, Richardson A, Pérez VI. Update on the oxidative stress theory of aging: does oxidative stress play a role in aging or healthy aging? Free Radic Biol Med. 2010 Mar;48(5):642-55.

3 Black CN, Bot M, Scheffer PG, Penninx BW. Sociodemographic and Lifestyle Determinants of Plasma Oxidative Stress Markers 8-OHdG and F2-Isoprostanes and Associations with Metabolic Syndrome. Oxid Med Cell Longev. 2016;2016:7530820.

4 Jones DP. Radical-free biology of oxidative stress. Am J Physiol Cell Physiol. 2008 Oct;295(4):C849-68.

5 Jansen EH, Beekhof PK, Viezeliene D, Muzakova V, Skalicky J. Long-term stability of oxidative stress biomarkers in human serum. Free Radic Res. 2017 Dec;51(11-12):970-7.

6 Frijhoff J, Winyard PG, Zarkovic N, Davies SS, Stocker R, Cheng D, et al.; Clinical Relevance of Biomarkers of Oxidative Stress. Clinical Relevance of Biomarkers of Oxidative Stress. Antioxid Redox Signal. 2015 Nov; 23(14):1144-70.

7 Schöttker B, Brenner H, Jansen EH, Gardiner J, Peasey A, Kubínová R, et al. Evidence for the free radical/ oxidative stress theory of ageing from the CHANCES consortium: a meta-analysis of individual participant data. BMC Med. 2015 Dec;13(1):300. 
8 Fernández-Sánchez A, Madrigal-Santillán E, Bautista M, Esquivel-Soto J, Morales-González A, Esquivel-Chirino C, et al. Inflammation, oxidative stress, and obesity. Int J Mol Sci. 2011;12(5):3117-32.

9 Furukawa S, Fujita T, Shimabukuro M, Iwaki M, Yamada Y, Nakajima Y, et al. Increased oxidative stress in obesity and its impact on metabolic syndrome. J Clin Invest. 2004 Dec;114(12):1752-61.

10 Poljsak B. Strategies for reducing or preventing the generation of oxidative stress. Oxid Med Cell Longev. 2011; 2011:194586.

11 Keaney JF Jr, Larson MG, Vasan RS, Wilson PW, Lipinska I, Corey D, et al.; Framingham Study. Obesity and systemic oxidative stress: clinical correlates of oxidative stress in the Framingham Study. Arterioscler Thromb Vasc Biol. 2003 Mar;23(3):434-9.

12 Vincent HK, Taylor AG. Biomarkers and potential mechanisms of obesity-induced oxidant stress in humans. Int J Obes. 2006 Mar;30(3):400-18.

13 Davì G, Guagnano MT, Ciabattoni G, Basili S, Falco A, Marinopiccoli M, et al. Platelet activation in obese women: role of inflammation and oxidant stress. JAMA. 2002 Oct;288(16):2008-14.

14 Aroor AR, DeMarco VG. Oxidative stress and obesity: the chicken or the egg? Diabetes. 2014 Jul;63(7):2216-8

15 Schöttker B, Haug U, Schomburg L, Köhrle J, Perna L, Müller H, et al. Strong associations of 25-hydroxyvitamin $\mathrm{D}$ concentrations with all-cause, cardiovascular, cancer, and respiratory disease mortality in a large cohort study. Am J Clin Nutr. 2013 Apr;97(4):782-93.

16 World Health Organization. Obesity: preventing and managing the global epidemic. Geneva: WHO; 2000.

17 National Institutes of Health, National Heart, Lung, and Blood Institute. Clinical guidelines on the identification, evaluation, and treatment of overweight and obesity in adults. The Evidence Report. NIH Publication No. 98-4083. Bethesda: NIH; 1998.

18 World Health Organization. Waist circumference and waist-hip ratio: Report of a WHO Expert Consultation. Geneva: WHO; 2008.

19 Schöttker B, Saum KU, Jansen EH, Boffetta P, Trichopoulou A, Holleczek B, et al. Oxidative stress markers and all-cause mortality at older age: a population-based cohort study. J Gerontol A Biol Sci Med Sci. 2015 Apr; $70(4): 518-24$.

20 Voorrips LE, Ravelli AC, Dongelmans PC, Deurenberg P, Van Staveren WA. A physical activity questionnaire for the elderly. Med Sci Sports Exerc. 1991 Aug;23(8):974-9.

21 Mukaka MM. Statistics corner: A guide to appropriate use of correlation coefficient in medical research. Malawi Med J. 2012 Sep;24(3):69-71.

22 Desquilbet L, Mariotti F. Dose-response analyses using restricted cubic spline functions in public health research. Stat Med. 2010 Apr;29(9):1037-57.

23 Reuter S, Gupta SC, Chaturvedi MM, Aggarwal BB. Oxidative stress, inflammation, and cancer: how are they linked? Free Radic Biol Med. 2010 Dec;49(11):1603-16.

24 Bredella MA. Sex Differences in Body Composition. Adv Exp Med Biol. 2017;1043:9-27.

25 Fujita K, Nishizawa H, Funahashi T, Shimomura I, Shimabukuro M. Systemic oxidative stress is associated with visceral fat accumulation and the metabolic syndrome. Circ J. 2006 Nov;70(11):1437-42.

26 Montuschi P, Barnes PJ, Roberts LJ 2nd. Isoprostanes: markers and mediators of oxidative stress. FASEB J. 2004 Dec;18(15):1791-800.

27 Chrysohoou C, Panagiotakos DB, Pitsavos C, Skoumas I, Papademetriou L, Economou M, et al. The implication of obesity on total antioxidant capacity in apparently healthy men and women: the ATTICA study. Nutr Metab Cardiovasc Dis. 2007 0ct;17(8):590-7.

28 Amirkhizi F, Siassi F, Djalali M, Foroushani AR. Evaluation of oxidative stress and total antioxidant capacity in women with general and abdominal adiposity. Obes Res Clin Pract. 2010 Jul-Sep;4(3):e163-246.

29 Suzuki K, Inoue T, Hioki R, Ochiai J, Kusuhara Y, Ichino N, et al. Association of abdominal obesity with decreased serum levels of carotenoids in a healthy Japanese population. Clin Nutr. 2006 Oct;25(5):780-9.

30 Seidell JC, Flegal KM. Assessing obesity: classification and epidemiology. Br Med Bull. 1997;53(2):238-52.

31 Ryo M, Maeda K, Onda T, Katashima M, Okumiya A, Nishida M, et al. A new simple method for the measurement of visceral fat accumulation by bioelectrical impedance. Diabetes Care. 2005 Feb;28(2):451-3.

32 Barinaga M. “Obese” protein slims mice. Science. 1995 Jul;269(5223):475-6.

33 Fonseca-Alaniz MH, Takada J, Alonso-Vale MI, Lima FB. Adipose tissue as an endocrine organ: from theory to practice. J Pediatr (Rio J). 2007 Nov;83(5 Suppl):S192-203.

34 Heilbronn LK, Ravussin E. Calorie restriction and aging: review of the literature and implications for studies in humans. Am J Clin Nutr. 2003 Sep;78(3):361-9. 